\title{
In vitro and in vivo investigation of bisphosphonate- loaded hydroxyapatite particles for peri-implant bone augmentation
}

\author{
Ulrike Kettenberger ${ }^{1}$, Vera Luginbuehl ${ }^{2}$, Philip Procter ${ }^{3}$ and Dominique P. Pioletti ${ }^{1 *}$ \\ ${ }^{1}$ Laboratory of Biomechanical Orthopaedics, Institute of Bioengineering, École Polytechnique Fédérale de Lausanne (EPFL), Switzerland \\ ${ }^{2}$ Pharmaceutical Technology, Institute of Biotechnology, Zürich University of Applied Sciences, Switzerland \\ ${ }^{3}$ Applied Materials Science, Department of Engineering Sciences, Uppsala University, Sweden
}

\begin{abstract}
Locally applied bisphosphonates, such as zoledronate, have been shown in several studies to inhibit peri-implant bone resorption and recently to enhance peri-implant bone formation. Studies have also demonstrated positive effects of hydroxyapatite (HA) particles on peri-implant bone regeneration and an enhancement of the anti-resorptive effect of bisphosphonates in the presence of calcium. In the present study, both hydroxyapatite nanoparticles (nHA) and zoledronate were combined to achieve a strong reinforcing effect on peri-implant bone. The nHA-zoledronate combination was first investigated in vitro with a pre-osteoclastic cell assay (RAW 264.7) and then in vivo in a rat model of postmenopausal osteoporosis. The in vitro study confirmed that the inhibitory effect of zoledronate on murine osteoclast precursor cells was enhanced by loading the drug on nHA. For the in vivo investigation, either zoledronate-loaded or pure nHA were integrated in hyaluronic acid hydrogel. The gels were injected in screw holes that had been predrilled in rat femoral condyles before the insertion of miniature screws. Micro-CT-based dynamic histomorphometry and histology revealed an unexpected rapid mineralization of the hydrogel in vivo through formation of granules, which served as scaffold for new bone formation. The delivery of zoledronate-loaded nHA further inhibited a degradation of the mineralized hydrogel as well as a resorption of the peri-implant bone as effectively as unbound zoledronate. Hyaluronic acid with zoledronate-loaded nHA, thanks to its dual effect on inducing a rapid mineralization and preventing resorption, is a promising versatile material for bone repair and augmentation. Copyright (c) 2015 John Wiley \& Sons, Ltd.
\end{abstract}

Received 30 April 2015; Revised 4 July 2015; Accepted 14 September 2015

Keywords drug delivery; hydrogel; hydroxyapatite; bisphosphonate; micro-CT; biomaterial mineralization

\section{Introduction}

Zoledronate-loaded hyaluronic acid hydrogel has recently been shown to efficiently improve early bone formation and inhibit bone resorption around an implant in a rat model of postmenopausal osteoporosis (Kettenberger et al., 2014). This is an important finding, as sufficient peri-implant bone density is directly linked to successful implant fixation (Stromsoe et al., 1993; Wilmes et al.,

*Correspondence to: Dominique P. Pioletti, Laboratory of Biomechanical Orthopaedics, Institute of Bioengineering, École Polytechnique Fédérale de Lausanne (EPFL), Station 19, 1015 Lausanne, Switzerland. E-mail: dominique.pioletti@epfl.ch
2006; Wirth et al., 2011). Reliable implant anchorage is, however, difficult to achieve in patients suffering from bone diseases that are characterized by a reduced bone density, such as osteoporosis. Implant migration and revision surgeries are often the consequence (Lobo-Escolar et al., 2010). Therefore, the local delivery of a bisphosphonate (BP) to the peri-implant bone stock, as demonstrated with the drug-loaded hydrogel (Kettenberger et al., 2014), is a promising approach to improve the outcome of implant fixation in patients presenting low bone quality.

In parallel, other strategies have been shown to successfully improve implant anchorage. One of them is the application of hydroxyapatite (HA) particles to the implant bed. Tami et al. (2009) demonstrated that HA particles that are 
filled into a screw hole are able to induce and maintain a denser bone mantle around the implant in osteoporotic bone. HA is chemically similar to the inorganic components of the bone matrix and therefore exhibits excellent biocompatibility with soft and hard tissues (Zhou and Lee, 2011). It is known to be bioactive, osteoconductive and biodegradable and is therefore widely used as a biomaterial for dental and orthopaedic applications (LeGeros, 2008). Considering also the fact that $\mathrm{Ca}^{2+}$ ions have been shown to enhance the inhibitory effect of nitrogen-containing BPs on osteoclast precursor cells by enhancing their endocytic internalization in vitro (Monkkonen et al., 1994; Thompson et al., 2006), it can be assumed that a combination of BPs and calcium phosphate particles may have enhanced effects on bone remodelling.

So far BPs have mainly been absorbed on HA bulk implants or coatings using the high affinity of the drug to minerals (Yoshinari et al., 2002; Peter et al., 2006; Suratwala et al., 2008; Faucheux et al., 2009; Sörensen et al., 2013). Binding BPs to bulk hydroxyapatite is associated with a slow release of the drug, as a low $\mathrm{pH}$ is required for a spontaneous release of the drug from a mineral surface (Rogers et al., 2000; Nancollas et al., 2006). However, as shown in our previous work, rapid availability of the BPs seems to be important for a positive effect on early bone formation (Kettenberger et al., 2014). The combination of BPs with small enough HA particles that could be distributed easily in bone and taken up by osteoclasts seems to be an advantageous idea.

Several studies have investigated the applicability of BP-loaded nanoparticles for antitumour drug-delivery properties (Palazzo et al., 2007) and for intravenous drug-delivery systems (Ong et al., 2008). Boanini et al. (2012) showed that zoledronate-containing HA nanocrystals promote osteoblast formation and osteoclast apoptosis in vitro, but they did not publish comparative results with pure zoledronate. Nejadnik et al. (2014) recently demonstrated that BP-functionalized hyaluronic acid containing calcium phosphate particles is stable in vivo and attracts bone ingrowth. However, for this study pamidronate was used only as a chemical link between calcium phosphate particles and the hydrogel matrix (Wang et al., 2006). In contrast to the present study, release of the drug to bone was not intended and was expected to be minimal (Nejadnik et al., 2014).

In this study we investigated the effect of zoledronate alone and in the presence of nHA particles on preosteoclastic macrophages in vitro. In a second step, we evaluated the influence of hyaluronic acid hydrogel loaded with nano-sized HA particles and zoledronate on peri-implant bone formation and resorption in an osteoporotic rat model.

\section{Materials and methods}

Zoledronate was purchased from Enzo Life Sciences (cat. no. ALX-430-153-0000, Enzo Life Sciences, Farmingdale,
USA). Hydroxyapatite powder (nHA) with a particle size of $\sim 200 \mathrm{~nm}$ was bought from Sigma (cat. no. 677418, Sigma-Aldrich, St. Louis, MO, USA). The nHA particles, showing a round shape (Figure 1), were heat-sterilized before use. Cell culture medium (CCM) for the in vitro studies was prepared by mixing Dulbecco's modified Eagle's medium (DMEM; cat. no. 41966-029, Gibco, Life Technologies, Carlsbad, CA, USA) with 10\% fetal bovine serum (FBS; Sigma-Aldrich, cat. no. F7524-500ML), 1\% penicillin-streptomycin (cat. no. 15140122, Gibco) and 1\% L-glutamine (cat. no. 25030024, Gibco).

\subsection{In vitro cell assay}

Murine macrophages (RAW 264.7, passages 13-16) were used for the present study, as cells from this line are regarded as osteoclastic precursors (Cuetara et al., 2006) and have been shown to react in vitro to BP exposure in a similar way to osteoclasts, and to well tolerate the presence of HA particles (Scheel et al., 2009; Abe et al., 2012).

An aqueous zoledronate solution with a concentration of $2 \mathrm{mg} / \mathrm{ml}$ was prepared and sterile-filtered. The drug solution was diluted with CCM to final concentrations of 10 and $0.625 \mu \mathrm{M}$ zoledronate (Zol assay). The dilution procedure was repeated with an aqueous dispersion containing $2 \mathrm{mg} / \mathrm{ml}$ zoledronate and $20 \mathrm{mg} / \mathrm{ml} \mathrm{nHA} \mathrm{(nHA-}$ Zol assay) and a dispersion containing only $20 \mathrm{mg} / \mathrm{ml}$ nHA (nHA assay).

Macrophages were seeded at a density of 2500 cells/well in 96-well plates (Microtest ${ }^{\mathrm{TM}}$ 96, Becton Dickinson Labware, NJ, USA), using six wells/group and incubated with $100 \mu \mathrm{l}$ CCM under standard incubation conditions $\left(37^{\circ} \mathrm{C}, 5 \% \mathrm{CO}_{2}\right)$. After $24 \mathrm{~h}$ of incubation, $100 \mu \mathrm{l}$ pure CCM or one of the six prepared CCMs containing zoledronate and/or nHA were added to the cells. Seven experimental groups were therefore included: one control group with cells exposed only to CCM; two groups exposed to 0.04 and $1.36 \mu \mathrm{g} / \mathrm{ml} \mathrm{nHA}$; two groups exposed to 0.16 and $5 \mu \mathrm{M}$ zoledronate; and two groups exposed to either $0.04 \mu \mathrm{g} / \mathrm{ml} \mathrm{nHA}$ loaded with $0.16 \mu \mathrm{M}$ zoledronate or $1.36 \mu \mathrm{g} / \mathrm{ml} \mathrm{nHA}$ loaded with $5 \mu \mathrm{M}$ zoledronate. A cell

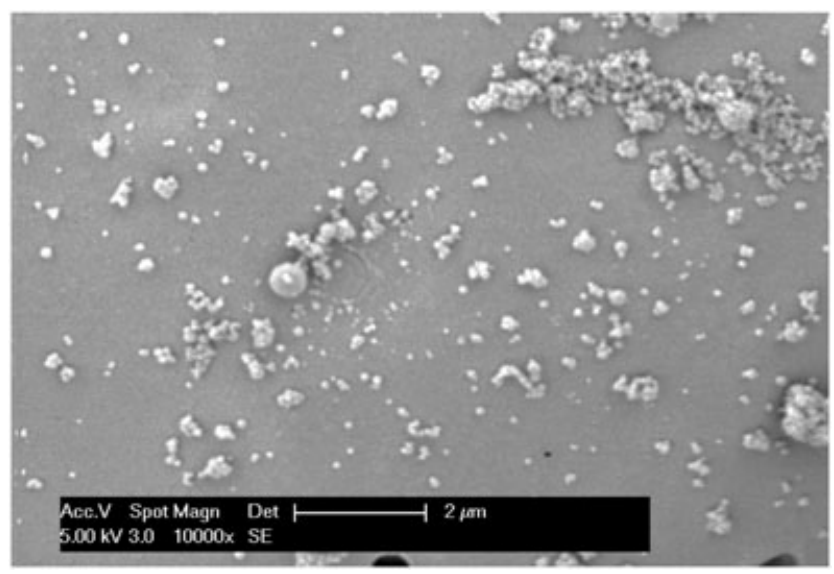

Figure 1. Scanning electron microscopy image of the hydroxyapatite nanoparticles used for the present study 
proliferation test (CellTitre $96 \AA$ AQueous One Solution, Promega, Fitchburg, WI, USA) was performed after 24, 48 and $72 \mathrm{~h}$ of cell exposure to the modified CCM. The absorbance was measured at $490 \mathrm{~nm}$ using a plate reader (Wallac 1420 Victor 2, Perkin-Elmer, Waltham, MA, USA). Background plates with modified media only were measured at all time points to avoid a bias caused by nHA particles in the dispersions. Light images of the cells were taken using an Axiovert 100 microscope equipped with an AxioCam MRc camera (both Carl Zeiss AG, Feldbach, Germany).

\subsection{Hydrogel preparation}

Commercially available cross-linked hyaluronic acid (Termira AuxiGel $^{\mathrm{TM}}$, Stockholm, Sweden) was used as the drug-delivery system for particles and zoledronate. This gel has already been shown to be a suitable drugdelivery system for BPs (Kettenberger et al., 2014). The preparation of the hydrogel was done under sterile conditions in a laminar flow hood. An aqueous solution containing $2 \mathrm{mg} / \mathrm{ml}$ zoledronate was prepared and sterile-filtered. nHA particles were added to the solution at a nHA:zoledronate ratio of 100:1; the exact drug dose was ensured by mixing precisely weighed and measured amounts of zoledronate, nHA and distilled water. Crosslinking of the hydrogel was achieved by mixing a hyaluronan derivative (component A) with a PVA crosslinker (component B); five parts of the zoledronate-nHA solution were mixed with three parts component $\mathrm{A}$ and two parts component B for the nHA-Zol-Gel group. The zoledronate-nHA dispersion was replaced by doubledistilled water with nHA for the nHA-Gel group. The resulting gels were allowed to settle for $1 \mathrm{~h}$ before filling the capillary pistons of a positive displacement pipette (Microman ${ }^{\circledR}$, Gilson, Middleton, USA) with $5 \mu$ l gel each. The capillaries were pulled off the pipette with the gel inside and left for $1 \mathrm{~h}$ for settling, then they were sterile-packed in plastic tubes and frozen at $-20^{\circ} \mathrm{C}$.

\subsection{In vivo study}

An in vivo study in a rat femoral model of postmenopausal osteoporosis was performed following a protocol that was established and published earlier by our group (Kettenberger et al., 2014). All animal procedures were approved by the local animal care and use committee (License No. 2508.1, EXPANIM, SCAV, Epalinges, Switzerland).

\subsubsection{Surgical procedures}

Eight virgin female Wistar rats (Janvier Labs, SaintBerthevin, France) were ovariectomized bilaterally using a dorsal approach (Alghamdi et al., 2013) at age 17 weeks and weight of $295 \pm 18 \mathrm{~g}$ to induce an oestrogen deficiency-related bone loss (Hogan et al., 2000). The rats were housed four/cage under $12 \mathrm{~h}$ light: $12 \mathrm{~h}$ dark cycles at $22{ }^{\circ} \mathrm{C}$ room temperature with $55 \%$ humidity. They were fed a standard rodent diet (Kliba Nafag 3436, Provimi Kliba AG, Switzerland) and tap water ad libitum. After ovariectomy (OVX) of the rats, food intake was limited to $50 \mathrm{~g} / \mathrm{kg}$ body weight/day. Sterilized hay, paper tunnels and wooden sticks were offered as cage environment enrichment. The animals were fed in groups, therefore an equal nutrition of all animals was ensured by close monitoring and regular weighing.

One miniature screw was implanted into each femoral condyle of 22 week-old rats with a mean weight of 350 $\pm 20 \mathrm{~g}$. The screws, with a thread length of $3 \mathrm{~mm}$ and a diameter of $1.4 \mathrm{~mm}$, were custom-made from radio-opaque polyetheretherketone (PEEK; RISystem, Davos, Switzerland) and coated with a $100 \mathrm{~nm}$ layer of titanium to mimic the interface with a standard orthopaedic screw. Before screw insertion, the predrilled unicortical screw holes (diameter $1.2 \mathrm{~mm}$, depth $3.5 \mathrm{~mm}$ ) were filled with $5 \mu \mathrm{l}$ of the prepared hydrogel containing only nHA (nHA-Gel group) or nHA and $5 \mu \mathrm{g}$ zoledronate (nHA-Zol-Gel group), using a positive displacement pipette. Four animals were assigned to each group, while both legs of each animal were treated with the same hydrogel to avoid an unwanted drug effect on the contralateral side.

\subsubsection{In vivo micro-CT imaging and image processing}

In vivo micro-CT scans (Skyscan 1076, Bruker micro-CT, Kontich, Belgium) of the right femur only were performed 1 day before OVX and 1 day before screw implantation, in order to confirm the bone loss caused by the oestrogen deficiency. Both femurs were then scanned at days 3, 10, 17, 31, 45 and 58 after screw implantation for the dynamic histomorphometry. The parameters for data acquisition and reconstruction were chosen according to our published protocol (Kettenberger et al., 2014). The animals were kept under isoflurane anaesthesia during the scanning time to avoid motion artifacts. All rats were euthanized at the time of the last micro-CT scan with an intraperitoneal injection of pentobarbital (Esconarkon, Strauli Pharma SA, Uznach) while under anaesthesia.

The image taken for evaluation of the bone loss and for dynamic histomorphometry were processed and analysed, using the software Amira ${ }^{\circledR}$ (FEI Vizualisation Sciences Group, Burlington, USA) and CTan (Bruker micro-CT, Kontich, Belgium), following a protocol published earlier (Kettenberger et al., 2014). Dynamic histomorphometry is an image-processing technique based on registration and comparison of consecutive micro-CT scans (Waarsing et al., 2004; Roshan-Ghias et al., 2011; Schulte et al., 2011). Mineralized tissue volume that was present only on the first micro-CT scan was considered to be resorbed/degraded. Mineralized tissue present on both scans was considered to be quiescent, and a mineralized tissue that could be found only on the second scan was considered to be newly mineralized tissue/formed bone. For an analysis of the spatio-temporal effect, the static 
and dynamic bone parameters were analysed in four layers of $368 \mu \mathrm{m}$, each located around the screw. This partition has been shown to furnish useful information about the peri-implant bone formation and resorption processes (Kettenberger et al., 2014).

The bone parameters from this study were compared with the results from animals that were treated with zoledronate-loaded hydrogel and pure hydrogel during a previous study (Kettenberger et al., 2014). This comparison was used to investigate synergistic effects between locally delivered nHA and zoledronate, as well as between hyaluronic acid hydrogel and nHA. Only one static parameter, mineralized tissue fraction (MV/TV), was analysed for this study. The MV/TV values were normalized to the first micro-CT scan (day 3 after screw implantation) to rule out a bias caused by the nHA that was visible already on micro-CT of day 3 in the nHA-Gel and he nHA-Zol-Gel groups. In contrast to our earlier publication, mineralization rate (bone formation rate) and demineralization rate (bone resorption rate) were defined in this study as newly mineralized (formed) and demineralized (resorbed) bone volume over total bone volume [over quiescent bone volume in the earlier publication (Kettenberger et al., 2014)] to be comparable with other studies.

\subsection{Histology}

The rat femurs were dissected immediately following euthanasia at week 58 after screw implantation and fixed, dehydrated and embedded in PMMA, following a previously published protocol (Kettenberger et al., 2014). After polymerization, the sections were cut, glued to PMMA slides and polished before etching their surface with $0.7 \%$ formic acid (Applichem, Gatersleben, Germany), then stained with Giemsa (VWR, Dietikon, Switzerland) or toluidine blue (Promega). Microscopy images for evaluation were taken using an upright microscope (DM 5500, Leica Microsystems, Wetzlar, Germany). The ground sections were finally X-rayed (Skyscan 1076) for tissue density mapping.

\subsection{Statistics}

Statistical testing was done using Matlab (Mathworks, Natick MA, USA). The Mann-Whitney U-test was used to evaluate statistical differences in the cell culture assays, as not all results were normally distributed. The static and dynamic mineralized tissue parameters were tested for significance using non-parametric Kruskal-Wallis ANOVA followed by a Tukey's honestly significant difference (HSD) test, since not all results followed a normal distribution and had an equal variance. Values lying outside an interval of 1.5 times the quartile range were identified as outliers and excluded when analysing the bone parameters.

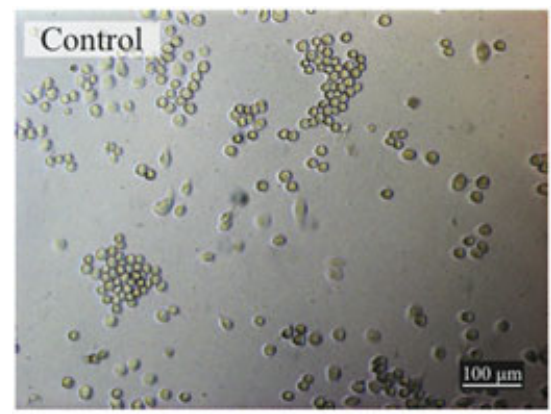

\section{Results}

\subsection{In vitro bioactivity of zoledronate-loaded $\mathrm{nHA}$ in osteoclastic precursor cells}

A visual inspection of the RAW 264.7 murine macrophages via microscopy revealed that these cells were
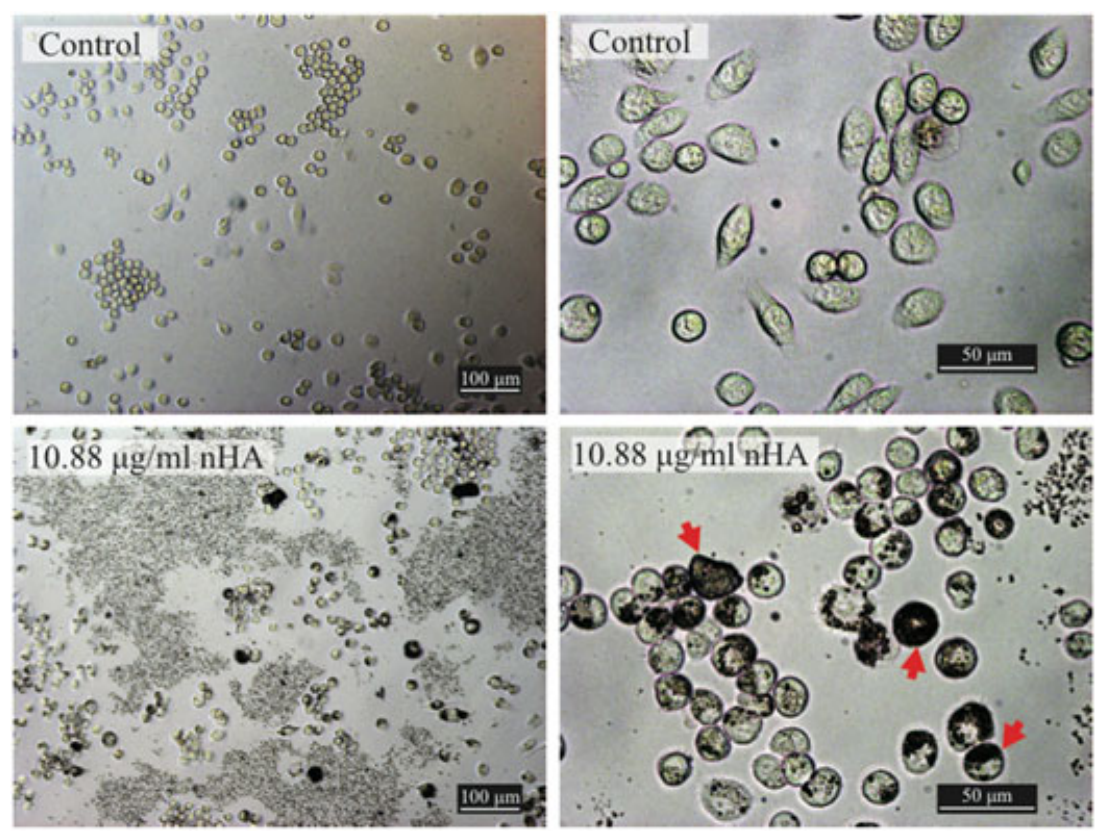

Figure 2. Light microscopy images of RAW 264.7 murine macrophages after $72 \mathrm{~h}$ of incubation with (bottom) and without (top) $\mathrm{nHA}$. The displayed cells were exposed to a particle concentration that was up to eight times higher than in the cell-culture assay. The macrophages were able to internalize large amounts of particles (red arrows); in this way they took up all the particles in their direct surroundings (bottom left). [Colour figure can be viewed at wileyonlinelibrary.com] 
capable of internalizing large amounts of the nanoparticles used in the present study, as they took up all particles in their direct surroundings (Figure 2). When looking at the proliferation of the macrophages incubated with different concentrations of nHA particles dispersed in the CCM, it can be seen that the nHA particles are well tolerated by the cells. No significant differences from the control group could be found for both tested concentrations at all three time points (Figure 3 ). When cultivated with either a high or a low dose of zoledronate, the RAW 264.7 macrophages showed a typical dose- and time-dependent proliferation decrease. This decrease was significant compared to untreated control cells only for the high zoledronate concentration $(5 \mu \mathrm{M})$ after 24 and $48 \mathrm{~h}$, and for both tested concentrations after $72 \mathrm{~h}$ $(p<0.05)$ (Figure 3$)$.

The dose- and time-dependent cell proliferation decrease was clearly enhanced in the two groups exposed to nHA loaded with zoledronate compared to the cells treated with zoledronate alone, indicating a clear

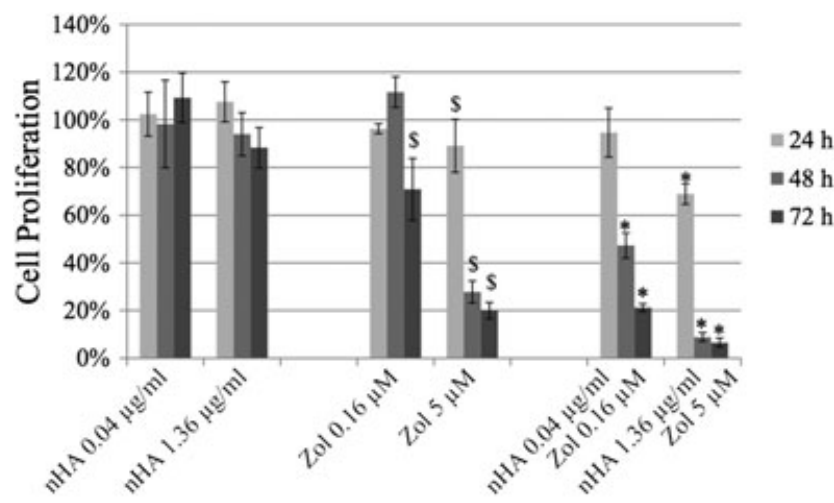

Figure 3. Proliferation study with RAW 264.7 macrophages incubated with two concentrations of hydroxyapatite particles (nHA), pure zoledronate (Zol) or zoledronate-loaded hydroxyapatite particles (nHA-Zol). The proliferation was measured after 24, 48 and $72 \mathrm{~h}$, using a cell titre assay. The proliferation of cells treated only with nHA particles did not differ significantly from the control cells at any concentration or time point. Significant differences between the zoledronate-treated cells (Zol) and control are marked $\left({ }_{p}^{\$}<0.05\right)$. Significant differences between the zoledronate-treated cells (Zol) and the corresponding cells treated with the same zoledronate concentration and nHA particles (nHA-Zol) are also marked (*p m 0.01) synergistic effect. The difference was statistically significant only for the high concentration $(5 \mu \mathrm{M})$ after $24 \mathrm{~h}$ and for both tested drug concentrations after 48 and $72 \mathrm{~h}(p<0.01)$ (Figure 3).

\subsection{In vivo study}

All rats tolerated both surgeries well and returned to normal activity right after surgery. The limited food intake led to a controlled weight gain of the animals; their final mean weight was $384 \pm 24 \mathrm{~g}$. Analysis of the bone parameters based on the micro-CT scans performed before and after ovariectomy confirmed a successful ovariectomy by showing a bone loss in all animals that was similar to the results found before in this model (Kettenberger et al., 2014) (data not shown).

\subsubsection{Micro-CT-based dynamic histomorphometry}

A total of 87 micro-CT scans were analysed for the dynamic histomorphometry, as three had to be excluded due to motion artefacts. One leg of one animal from the nHA-Zol-Gel group showed excessive bone formation; the bone parameters from this leg were identified as outliers and excluded. Five to eight samples/group and for each time point were left after removal of the outliers. The rapidity of the appearance and the structure of the mineralizing tissue that appeared on the micro-CT scans over time suggested that it could not be entirely attributed to formed bone (Figure 4). Histology confirmed later that the hydrogel-nHA composite became mineralized in vivo. As it was not possible to distinguish on the micro-CT scans between bone and mineralized biomaterial, we decided to analyse the mineralization (MR) and demineralization (DR) rates instead of bone formation and bone resorption rates, and mineralized tissue volume (MV) instead of bone volume (BV).

\subsubsection{Static parameters}

The results from this study were compared with the results from a previous study, where the hyaluronic acid hydrogel was implanted either pure (Gel group) or loaded only with zoledronate but no particles (Zol-Gel group)

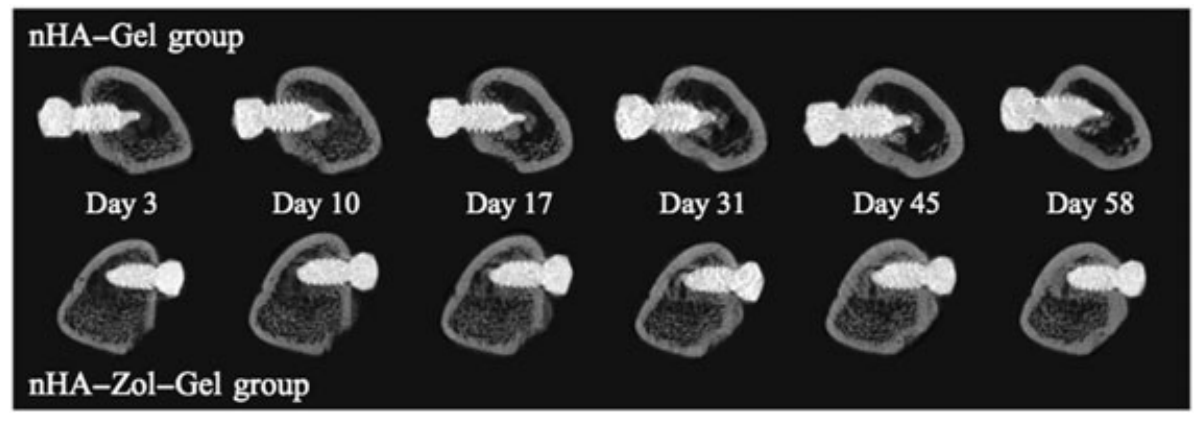

Figure 4. Micro-CT scans taken at six time points after surgery from the nHA-Gel group (top) and the nHA-Zol-Gel group (bottom). In both groups the images show a rapid mineralization of the tissue around the screws, which is resorbed and remodelled quickly in the nHA-Gel group and persists in the nHA-Zol-Gel group 
(Kettenberger et al., 2014). The analysis of the MV/TV in the layer next to the screw surface $(0-368 \mu \mathrm{m})$ showed no difference at any time between the nHA-Zol-Gel and Zol-Gel groups (Figure 5). In the second layer $(368-736 \mu \mathrm{m})$ the MV/TV gain was around 50\% higher in the nHA-Zol-Gel than the Zol-Gel group, starting from day 17. However, a large standard deviation (SD) prevented statistical significance of the results. This difference diminished to around $30 \%$ in the third layer $(736-1104 \mu \mathrm{m})$; again, a significant difference could not be shown, due to high variability of the results. No difference at all was visible in the fourth layer (1104-1472 $\mu \mathrm{m})$.

Close to the screw $(0-368 \mu \mathrm{m})$ the nHA in the hydrogel caused a mineralized tissue fraction gain that was around $30 \%$ higher than in the Gel group during the full experimental period. This difference, however, was not statistically significant. In the second layer $(368-736 \mu \mathrm{m})$, the MV/TV doubled between days 3 and 10, a gain comparable to the zoledronate-treated groups and significantly higher than in the Gel group, which showed a constant MV/TV. The difference between the nHA-Gel and Gel groups diminished later in the study and was only marginal at the end of the experimental period. A similar development could be observed in the third layer, where the initial gain in MV/TV was only $50 \%$ and the difference between the nHA-Gel and Gel groups equalled out by day 17. No difference between the nHA-Gel and Gel groups was found in the outermost layer (1104-1472 $\mu \mathrm{m})$. No other static bone parameters were analysed for this study, as all other typical bone parameters would be misleading when looking at mixture of bone and mineralizing biomaterial.

\subsubsection{Dynamic parameters}

The analysis of the dynamic parameters gives information about bone formation and resorption processes. In the
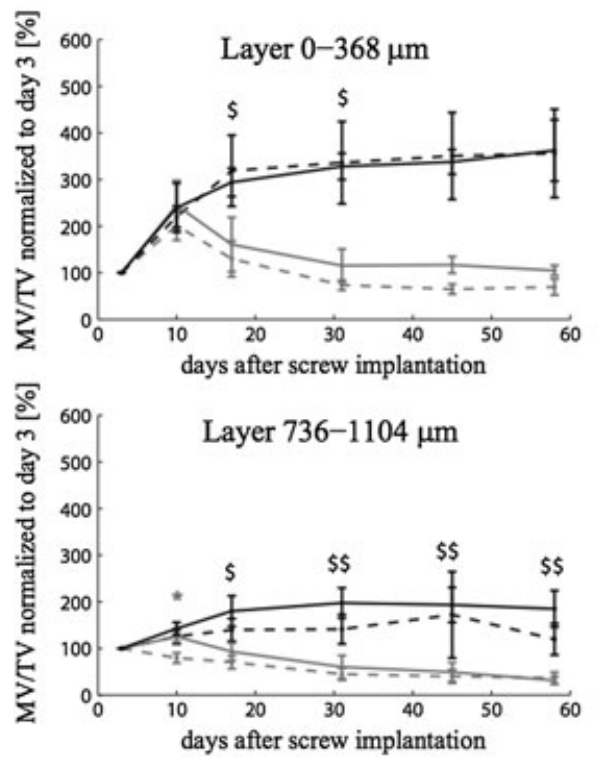

present study, it was not clear which part of the appearing mineralized structure could be attributed to new bone formation and which part to mineralizing biomaterial. Therefore, we measured a general mineralization rate instead of a bone formation rate, and a demineralization rate including bone resorption and biomaterial degradation. Three-dimensional (3D) volume rendering of the comparison of two consecutive micro-CT scans was used to get an overview of the mineralizing and demineralizing processes (Figure 6). Both experimental groups showed a significant mineralization around the screw between days 3 and 10, which was followed by a pronounced demineralization in the nHA-Gel group. The bone further away from the screw implantation site was also gradually resorbed in this group. In contrast, the nHA-Zol-Gel group showed only very little demineralization in the region analysed.

These findings were also represented in the measured mineralization and demineralization rates (Figure 7 and 8). No differences in the mineralization rate in direct contact with the screw (layer 0-368 $\mu \mathrm{m}$ ) could be found between the two groups of this study and the two groups without nHA from our previous study. In the second layer $(368-736 \mu \mathrm{m})$, however, between days 3 and 10 there was a highly significant difference between the nHA-Gel and Gel groups and a small trend to a higher mineralization rate in the nHA-Zol-Gel group compared to the group that released the drug directly from the hydrogel (Zol-Gel group). These differences equalled out by day 10 . The same situation could be found also in the two outer layers (736-1472 $\mu \mathrm{m})$.

A high bone resorption/demineralization was found between days 10 and 31, which is characteristic for the drug-free groups. This phenomenon was completely inhibited by the locally delivered zoledronate (Figure 8). The demineralization rate of the nHA-Zol-Gel group did not differ at any time and in any region from that of the Zol-Gel group. When comparing the nHA-Gel and Gel
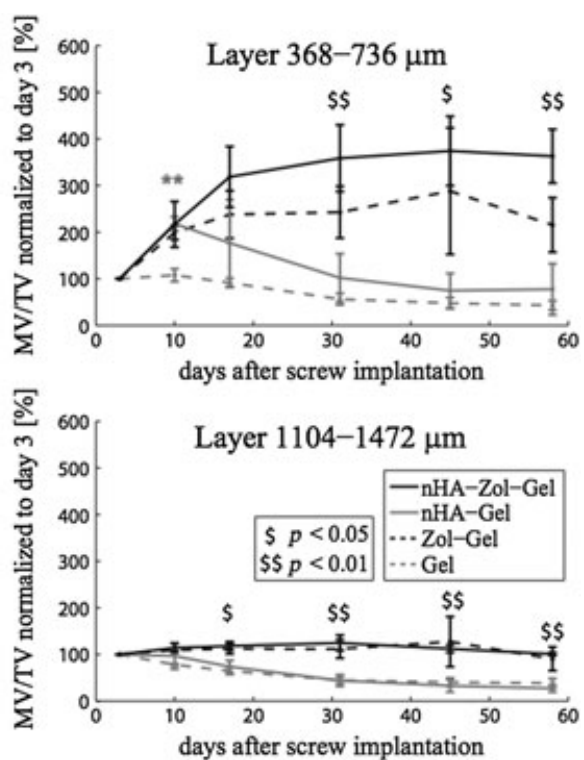

Figure 5. Mineralized volume/tissue volume (MV/TV) measured in four layers around the screw: *significant differences between the nHA-Gel and Gel groups; \$significant differences between the nHA-Zol-Gel and nHA-Gel groups; data from the Zol-Gel and Gel groups were acquired during an earlier study [1] and are shown for comparison 


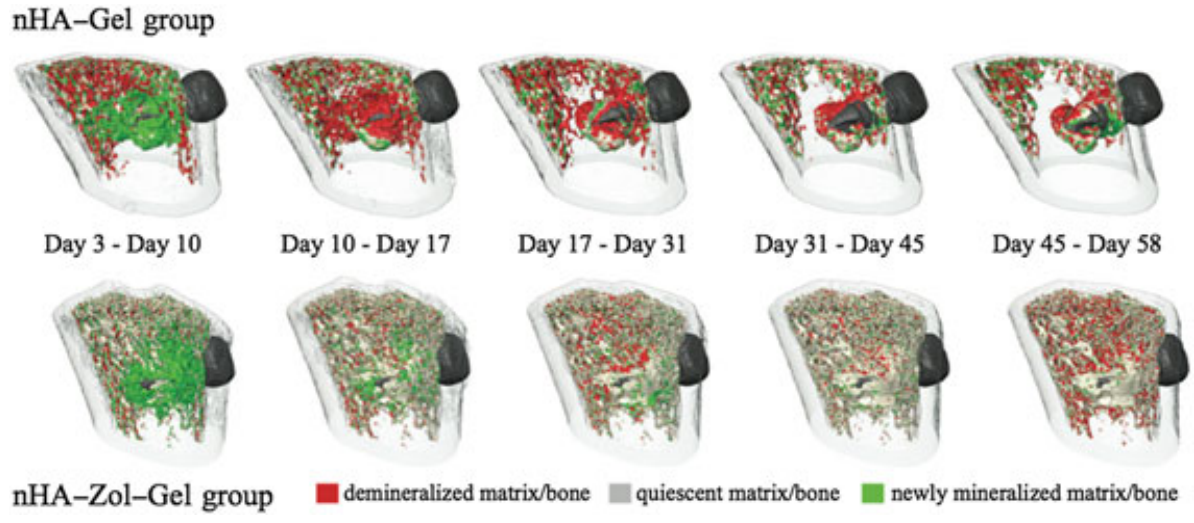

Figure 6. 3D visualization of a comparison of two consecutive micro-CT scans: two representative samples were taken, one from the nHA-Gel group (top) and one from the Zol-Gel group (bottom); the analysed trabecular region of the bone is shown in light grey, red and green, and the screw in dark grey; the cortex is transparent. [Colour figure can be viewed at wileyonlinelibrary.com]
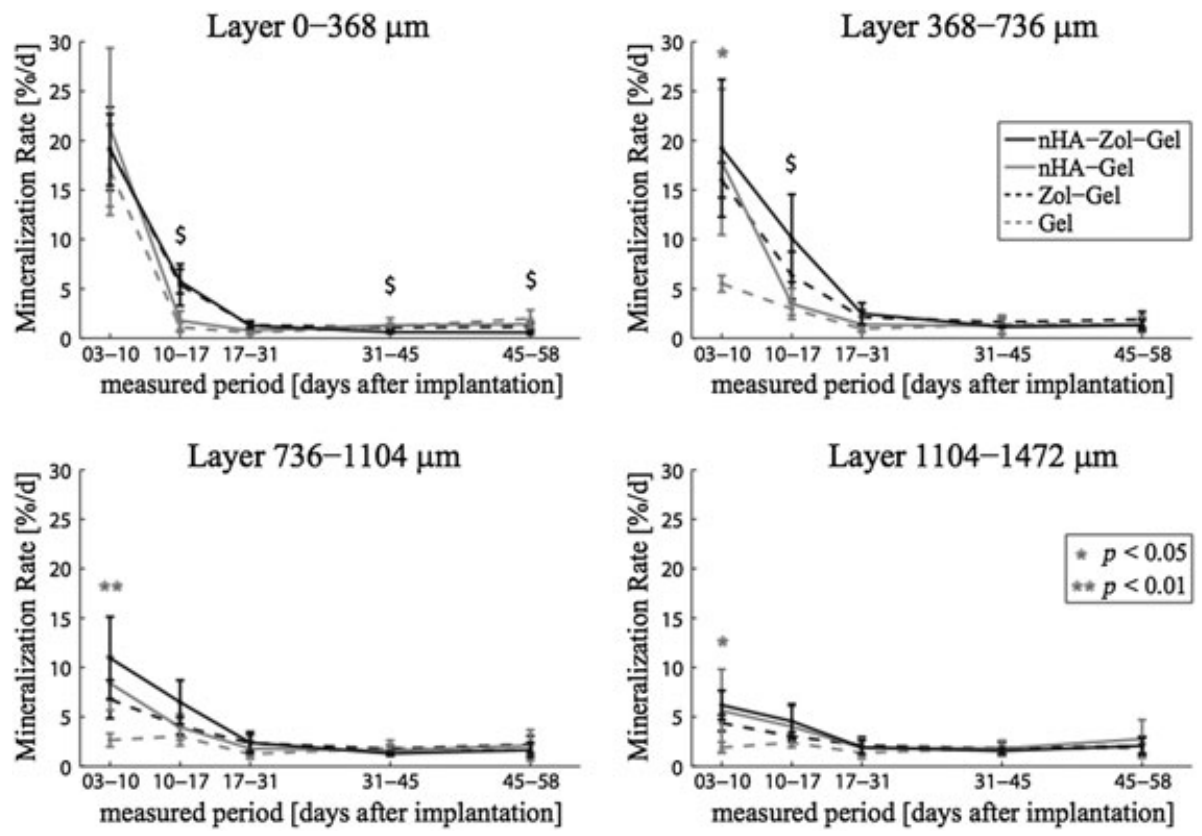

Figure 7. Mineralization rate (MR), including bone formation and biomaterial mineralization, measured in four layers around the screw: "significant differences between the nHA-Gel and Gel groups; no significant differences were found between the nHA-Zol-Gel and nHA-Gel groups; data from the Zol-Gel and Gel groups were acquired during an earlier study [1] and are shown only for comparison

groups, there was an unexpected trend to a higher demineralization rate in the nHA-Gel group in the outer two layers $(736-1472 \mu \mathrm{m})$.

\subsection{Histology}

The histological results showed that the hyaluronic acid hydrogel is fully degraded after 58 days of implantation without leaving any visible residues (Figures 9, 10). All screws showed good osteo-integration without any inflammatory reaction or fibrotic tissue encapsulation. In both the nHA-Gel and nHA-Zol-Gel groups, highly mineralized regions were detected within the bone. These granule-shaped spots had a diameter of up to $50 \mu \mathrm{m}$ in the nHA-Gel group and extended across wide areas in the
nHA-Zol-Gel group. The X-rays taken of the histology slides indicated that these granules had a density comparable to/higher than bone. It can be excluded that those regions simply represented remaining nHA particles, as the inserted particles had a diameter of $<200 \mathrm{~nm}$ and the observed granules were much larger.

In the nHA-Gel group, only small amounts of these mineralized spots were found in islands of new bone located mainly close to the screw tip, the region where the hydrogel accumulates during screw insertion. The granules were completely embedded in the bone matrix and the resulting constructs showed a trabecular structure. No new bone formation could be detected in this group besides a thin layer close to the screw surface and the bone close to the mineralized biomaterial. Almost no trabecular bone was left around the screw in the nHA-Gel group 13 weeks after OVX. 

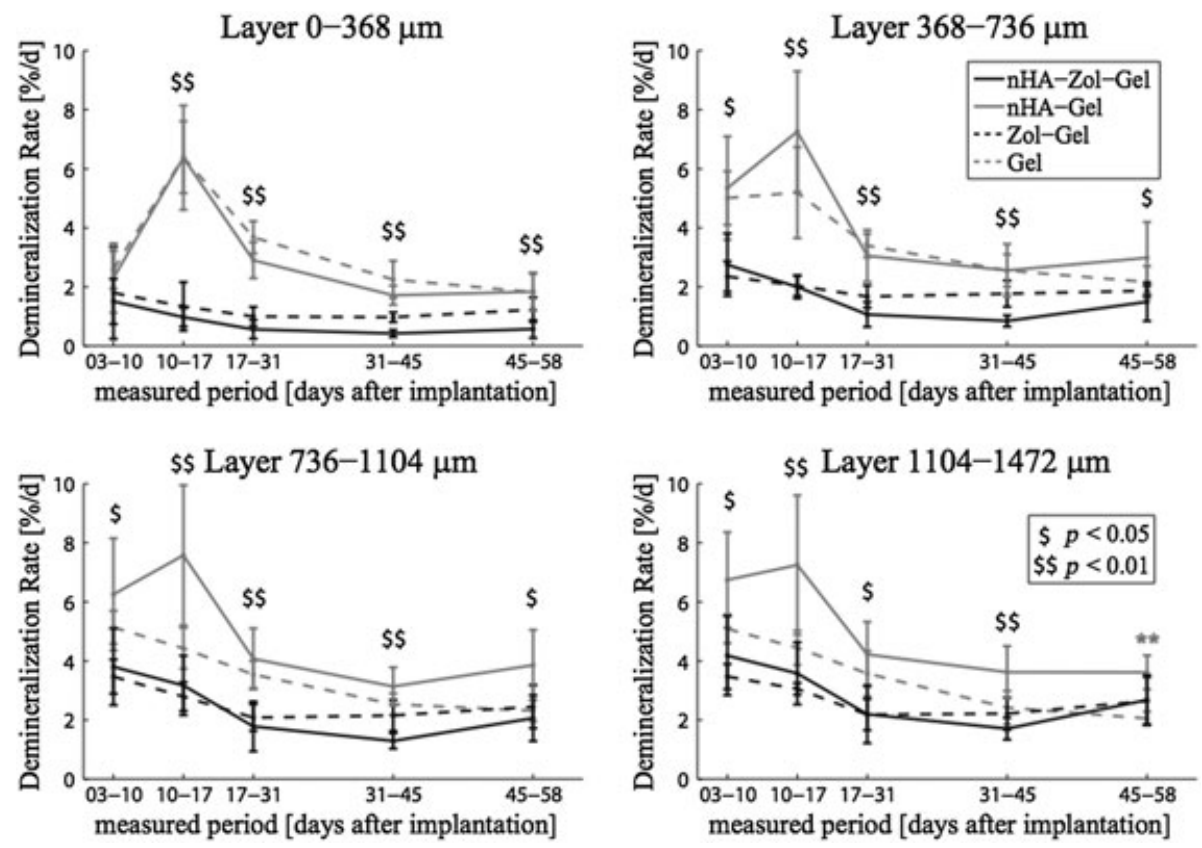

Figure 8. Demineralization rate (DR), including bone resorption and biomaterial degradation, measured in four layers around the screw: "significant differences between the nHA-Gel and Gel groups; $\$$ significant differences between the nHA-Zol-Gel and nHA-Gel groups; data from the Zol-Gel and Gel groups were acquired during an earlier study [1] and are shown only for comparison
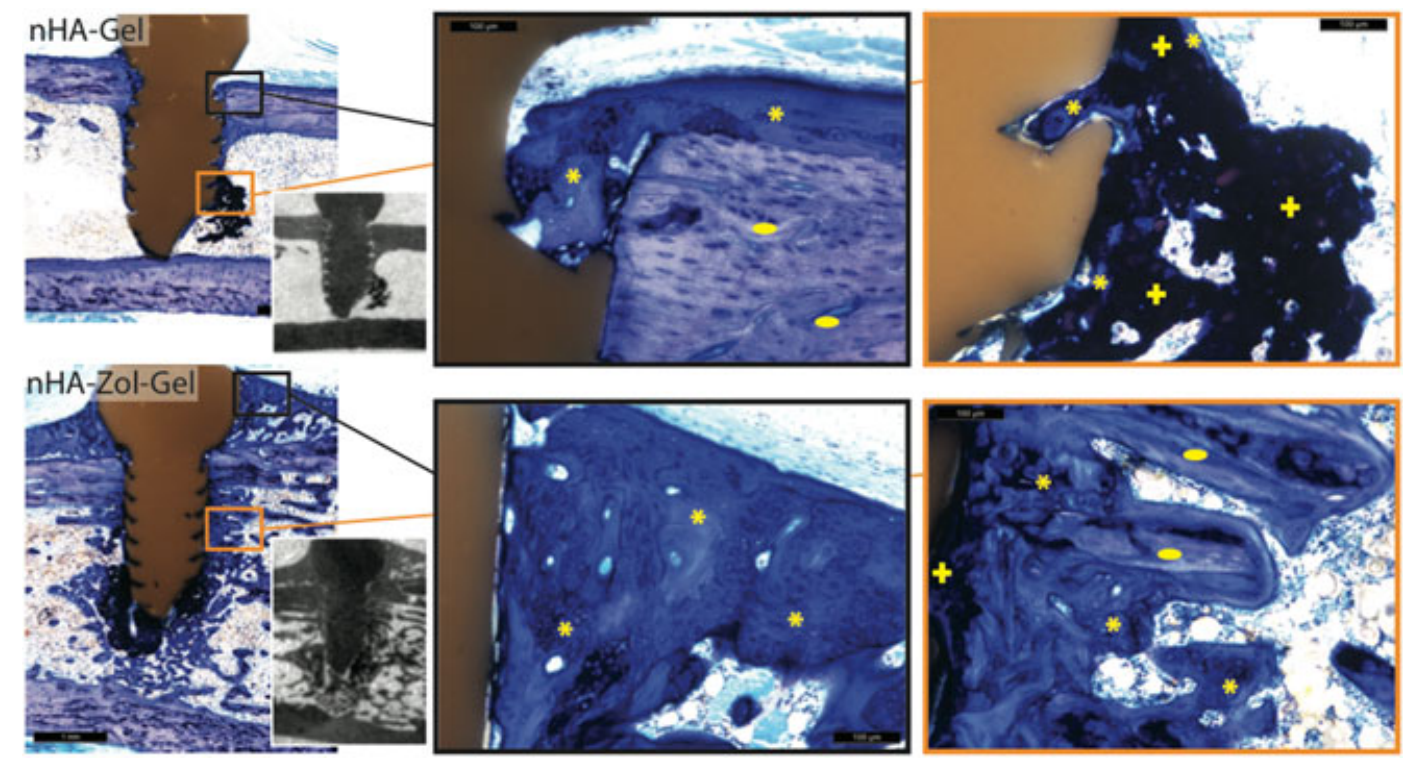

Figure 9. Toluidine blue-stained ground sections from the nHA-Gel (top) and nHA-Zol-Gel (bottom) groups: * (yellow) in the darker blue regions indicate immature, non-remodelled bone; ellipses (yellow) on brighter blue regions mark remaining old bone; mineralized hydrogel residues are highlighted with yellow crosses. The small black-and-white panels are X-rays of the histology slides and show the mineralization of each region. [Colour figure can be viewed at wileyonlinelibrary.com]

The nHA-Zol-Gel group showed a completely different situation. Significantly larger mineralized regions could be found, mainly close to the screw tip but also between the threads. The remaining mineralized biomaterial had a porous structure and was partly penetrated by bone tissue. In contrast to what was seen in the nHA-Gel group, the biomaterial-bone constructs were rather compact and did not show a trabeculae-like structure. In general, much more trabecular bone could be found both close to and further away from the screw in the nHA-Zol-Gel group compared to the nHA-Gel group. New bone was formed also at the interface to the mineralized hydrogel. A formation of large periosteal calli was found in the nHA-Zol-Gel group that was not present to this extent in the nHA-Gel group.

\section{Discussion}

The main goal of the present study was to investigate the bone-reinforcing effect of hydrogel loaded with both 


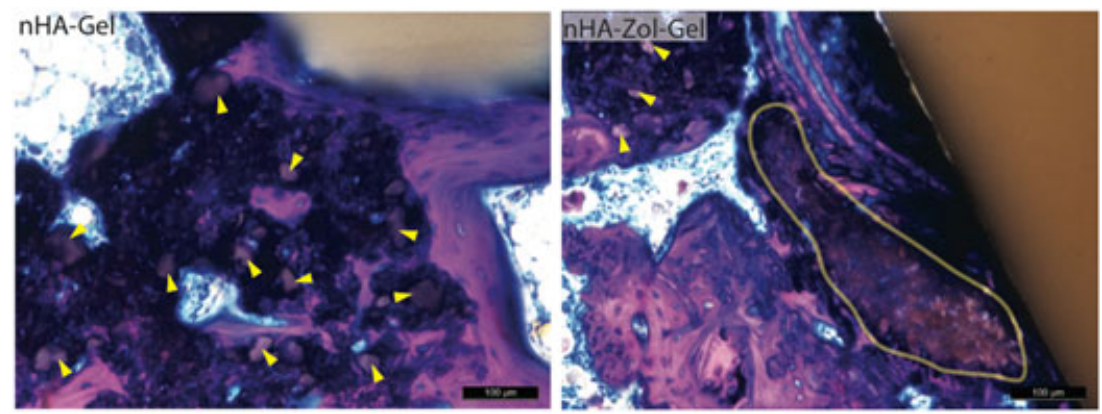

Figure 10. Details from the samples shown in Figure 9. The sections were stained with Giemsa for a better differentiation between bone (pink) and mineralized hydrogel (pink-brownish): (left) small granule-shaped mineralized spots from the nHA-Gel group are marked with yellow arrows; (right) an extended mineralized region from the nHA-Zol-Gel group is marked with a yellow line; smaller regions are also highlighted with yellow arrows. [Colour figure can be viewed at wileyonlinelibrary.com]

hydroxyapatite nanoparticles and the bisphosphonate zoledronate. An enhanced effect compared to the application of only one of the two substances was presumed, based on studies that demonstrated enhanced periimplant bone density after the application of HA particles (Tami et al., 2009). Furthermore, increased zoledronate potency in the presence of $\mathrm{Ca}^{+}$ions has been shown, as well as a favourable bone ingrowth in BP-linked hydrogel containing calcium phosphate particles (Monkkonen et al., 1994; Merrell et al., 2007; Nejadnik et al., 2014).

A cell proliferation assay that was performed with murine pre-osteoclastic RAW 264.7 macrophages showed a dose-dependent proliferation decrease for cells exposed to zoledronate. This result confirms the findings of Abe et al. (2012), who showed that nitrogen-containing BPs are cytotoxic for RAW 264.7 cells. The inhibitory effect of zoledronate was significantly enhanced by the presence of nHA particles, which were added to the solution at a nHA:zoledronate ratio of 10:1, indicating a clear synergistic effect between the two compounds. Due to the high affinity of zoledronate to hydroxyapatite, it must be assumed that most of the zoledronate was absorbed to the particles and therefore not present in solution (Nancollas et al., 2006). Light microscopy images showed that the macrophages internalized the zoledronate-loaded particles surrounding them. This finding suggests that the cells had taken up the drug together with the nHA particles. Thompson et al. (2006) showed that amino-BPs are typically internalized by macrophages and osteoclasts via fluid-phase endocytosis (pinocytosis) in vitro. Particles, however, have been shown to be incorporated by macrophages via pinocytosis only for sizes $<500 \mathrm{~nm}$; larger particles are phagocytosed (Kruth et al., 2005; Oh and Park, 2014). The particles used for this study had a size of $<200 \mathrm{~nm}$ but formed agglomerations, so that both mechanisms have to be considered. A stronger inhibitory effect on macrophages has also been described for BP-modified gold nanoparticles (size $<100 \mathrm{~nm}$ ) compared to unbound BP (Fanord et al., 2011). One possible explanation is a more specific intracellular accumulation of drug molecules at the site of action with the particles as carrier. In our study, an alternative explanation could be an enhancement of the BP potency by the presence of calcium, a phenomenon that has been demonstrated previously (Thompson et al., 2006). The $\mathrm{Ca}^{+}$ions could originate from hydroxyapatite digestion by lysosomal enzymes in the macrophages (Bloebaum et al., 1998). In order to rule out a negative effect of the nHA powder itself on the macrophages, we also cultured them only with particles. The results showed that the cells tolerated the presence of the particles well, a finding that has been published previously (Scheel et al., 2009).

An unexpected original finding of this study was the rapid in vivo mineralization of the hydrogel, which happened independently of the BP in both experimental groups. It is known that calcium phosphate particles can act as nucleation sites in hydrogel matrices, promoting HA precipitation (Gkioni et al., 2010). This phenomenon has been investigated mainly in vitro with simulated body fluids (Chen et al., 2011). We could not find any publications showing such a rapid mineralization in vivo. Nejadnik et al. (2014) recently published a study investigating the use of pure and BP-modified hyaluronic acid with calcium phosphate nanoparticles for bone repair. Despite a mineral content of the gel in the same range ( $6 \mathrm{vs} 10 \mathrm{w} / \mathrm{v} \%$ in the present study), they did not report a considerable mineralization of the hydrogels after 4 weeks of implantation in bony defects created in rat tibiae. However, in their study newly formed bone was analysed based only on histology. Depending on the staining used, histology might not be the most appropriate technique to detect biomaterial mineralization.

The highly mineralized spots that were found in the present study mainly showed a granule-like structure with a particle size of up to $50 \mu \mathrm{m}$, which supports the concept that they were formed by hydroxyapatite deposition, starting from the nHA particles as nucleation points. In the nHA-Zol-Gel group, the granules seemed even to have fused to larger mineralized regions, as shown by histology. A complete integration of the newly formed granules into the bone matrix without any signs of inflammation or foreign body reaction confirmed their excellent biocompatibility and osteoconductivity. When comparing the nHA-Gel group of this study with the Gel group of a former study, significant differences in early mineralization could be detected. The explanation for this phenomenon is an initial rapid mineralization of the biomaterial, followed by its simultaneously occurring degradation 
and penetration by bone tissue, as shown by structural changes on the time-lapse micro-CT scans and the terminal histology results. Lower amounts of residual mineralized spots in the nHA-Gel group compared to the nHA-Zol-Gel group indicate an efficient biodegradation of the HA. Similar findings have been published by Arts et al. (2006), who demonstrated that HA nano-powder is integrated in newly formed bone and efficiently recruits macrophages and osteoblasts in vivo.

The obvious mineralization of the biomaterial complicated evaluation of the drug effect on peri-implant bone. In vivo micro-CT imaging did not allow a differentiation between newly formed woven bone and mineralized hydrogel. When comparing the mineralized tissue volume fraction and the mineralization rate of the nHA-Zol-Gel group to the Zol-Gel group of an earlier study (Kettenberger et al., 2014), no significant differences could be detected at any time. However, there was a strong trend to more tissue mineralization that appeared early and remained visible during the whole experimental phase. Contributors to this gain of mineralized tissue could have been, on the one hand, new woven bone formation and, on the other hand, biomaterial mineralization. In our earlier study we stated that the discovered boost in early peri-implant bone formation was caused by an initial 'flush' of all bone cells with BP (Kettenberger et al., 2014). We did not investigate the release of particles from the gel, as it is highly dependent on the shape, swelling state and surrounding tissue of the hydrogel and is therefore difficult to mimic in vitro. However, it can be assumed that the release of the comparatively huge nHA particles is significantly slower than that of the small drug molecules. Orriss et al. (2009) showed in vitro that an inhibitory effect on osteoblast mineralization of bound zoledronate occurred at 10-100-fold higher concentrations compared to free zoledronate. As the non-phagocytic osteoblasts are unlikely to incorporate zoledronate-loaded particles, less influence on bone formation can be assumed for the nHA-Zol-Gel group compared to the situation when zoledronate is delivered from pure hydrogel (Zol-Gel group). This theory is also supported by the findings of Arslan et al. (2011), who could not find a significant influence of alendronate-soaked HA granules on bone formation compared to saline-soaked HA in a OVX mandibular defect model. Nevertheless, small but still significant differences could also be shown for the mineralization rate between the nHA-Gel and nHA-Zol-Gel groups. As a consequence, the zoledronate-nHA combination must influence bone formation and/or biomaterial mineralization in a different way from pure nHA; however, the mechanism is not clear.

When looking at the demineralization rate, the strong inhibitory effect of zoledronate becomes obvious in the highly significant differences between the nHA-Gel and nHA-Zol-Gel groups. The micro-CT study and histology revealed that the drug not only inhibited bone resorption but also biomaterial degradation, as can be seen by the absence of macrophages and giant foreign body cells and much larger amounts of mineralized material remaining in the nHA-Zol-Gel group. Histology also showed that the bone-biomaterial construct in the nHA-Gel group had already been remodelled to a trabecular structure, whereas it was still rather compact in the nHA-Zol-Gel group. There was no difference in the demineralization rate between the Zol-Gel group from the former study and the nHA-Zol-Gel group from the present study, even further away from the screw. Therefore, there must be either a release of the drug-loaded particles or a release of the drug alone, as it can be caused by the resorptive activity of surviving osteoclasts or macrophages (Coxon et al., 2006). Unfortunately, the present study could not give any answer to the question of by which means and how far the drug-loaded particles move in the bone environment in vivo.

Micro-CT-based histomorphometry was chosen for the present study as we found it to be a very suitable technique for the long-term 3D analysis of peri-implant bone remodelling (Kettenberger et al., 2014). The unexpected rapid mineralization found in the present study, however, interfered with the bone analysis, which is one inherent limitation of this study. The differentiation between bone and biomaterial can only be done by means of histology, which was scheduled for this study only at the end of the experimental period of 8 weeks. Future studies should therefore include more groups of animals so that histology can be performed at earlier time points for an observation of synergies between bone formation and biomaterial mineralization. More experiments are also needed to understand the exact mineralization processes and the composition and mechanical competence of the resulting mineral. The in vivo mineralization seems to be dependent on size, shape and material of the particles, as well as on type, density and degradation profile of the hydrogel, as similar studies with comparable materials did not report an in vivo mineralization (Nageeb et al., 2012; Martínez-Álvarez et al., 2013; Heo et al., 2014; Nejadnik et al., 2014). An investigation of the mechanical properties of the resulting material, as could be done by nano-indentation, will be key when it comes to an evaluation of implant stability. Another limitation of this study was the fact that the rats received the same material in both femoral condyles. Ideally, two different materials are implanted in one animal to improve the inter-sample variability; however, this was not done in the present study, in order to avoid a systemic effect of zoledronate on the contralateral side.

The hydrogels containing pure or zoledronate-loaded nHA particles were both easy to apply to bone, due to their soft consistency, which makes them ideal for an application to irregular-shaped cavities such as an implant bed or difficult-to-access bone defects. The indications for both materials, however, should still not be the same. The nHA-loaded hydrogel induced some new bone formation within the biomaterial in the present study, but was not able to stabilize the bone loss characteristic for OVX rats (Kettenberger et al., 2014). The nHA-loaded hydrogel could therefore have more potential in the repair and 
sealing of bony defects in healthy bone. The hydrogel with $\mathrm{nHA}$ and zoledronate, however, could be favourable for the repair of bone defects that require at the same time a preservation of the surrounding bone matrix. Attention has to be paid in this case to the drug dose and the density of the resulting mineralized spots. As zoledronate has a 'protective' effect for the biomaterial and inhibits its degradation, there is a risk that penetration by the bone tissue can become impossible if the biomaterial densifies too much and cannot be resorbed.

Despite the synergistic effects between hydroxyapatite nanoparticles and zoledronate that we were able to show in vitro, it was not possible to answer the question of whether the use of a nHA-zoledronate hydrogel further improves implant fixation compared to a hydrogel loaded only with zoledronate (Kettenberger et al., 2015). There was a non-significant increase of the mineralized tissue volume fraction in an extended range around the implant, but large amounts of biomaterial residues with unknown mechanical properties require mechanical testing before drawing a final conclusion. For the moment we can only conclude that both tested materials seem to be good candidates for bone repair in different specific clinical situations.

\section{Conclusion}

The present study was able to show a synergistic effect between zoledronate and nHA particles in vitro, as the inhibitory effect of the drug on osteoclast precursors was enhanced when administering zoledronate absorbed on hydroxyapatite nanoparticles. Furthermore, it could be demonstrated that nano-sized hydroxyapatite can serve as nucleation points in hyaluronic acid hydrogel in vivo in a rat model of postmenopausal osteoporosis. The material is then rapidly mineralizing under the formation of $\mu \mathrm{m}$-sized granules. These highly mineralized spots have been shown to be highly biocompatible, biodegradable and to serve as scaffolds for new bone formation. The biomaterial mineralization also occurred when zoledronate was absorbed to the nanoparticles before integrating them into the hydrogel. In this case, however, the forming granules were protected from degradation by the antiresorptive effect of the BP resulting in larger mineralized regions compared to drug-free nHA-hydrogel. At the same time, zoledronate was still active and efficiently inhibited the resorption of peri-implant bone to a similar extent to pure hydrogel loaded only with the drug.

\section{Acknowledgements}

Special thanks to Sandra Jaccoud at the LBO for her assistance during surgery and cell-culture experiments, as well as for the preparation of the histology slides. Special thanks also to Dr Eric Thein from CHUV for his assistance during surgery and to Professor Brigitte von Rechenberg, Dr Salim Darwiche and Dr Karina Klein from the University of Zürich for interpretation of the histology slides. This study was partially supported by (Kommission für Technologie und Innovation KTI, Switzerland; Grant No. 11098.1).

\section{References}

Abe K, Yoshimura Y, Deyama Y et al. 2012; Effects of bisphosphonates on osteoclastogenesis in RAW264.7 cells. Int $J$ Mol Med 29: 1007-1015.

Alghamdi HS, Bosco R, van den Beucken JJJP et al. 2013; Osteogenicity of titanium implants coated with calcium phosphate or collagen type-I in osteoporotic rats. Biomaterials 34: 3747-3757.

Arslan A, Altundal H, Cevik O et al. 2011; Comparison of the effects of local application of hydroxyapatite graft soaked with alendronate solution and pure hydroxyapatite graft in the mandible of ovariectomized rats. Biotechno Biotechnolog Equip 25: 2513-2518.

Arts JJC, Verdonschot N, Schreurs BW et al. 2006; The use of a bioresorbable nanocrystalline hydroxyapatite paste in acetabular bone impaction grafting. Biomaterials 27: $1110-1118$

Bloebaum RD, Lundeen GA, Bachus KN et al. 1998; Dissolution of particulate hydroxyapatite in a macrophage organelle model. J Biomed Mater Res 40: 104-114.

Boanini E, Torricelli P, Gazzano M et al. 2012; The effect of zoledronate-hydroxyapatite nanocomposites on osteoclasts and osteoblast-like cells in vitro. Biomaterials 33: 722-730.
Chen X, Meng Y, Wang Y et al. 2011; A biomimetic material with a high bio-responsibility for bone reconstruction and tissue engineering. J Biomater Sci Polym Edn 22: 153-163.

Coxon FP, Thompson K, Rogers MJ. 2006, Recent advances in understanding the mechanism of action of bisphosphonates. Curr Opin Pharmacol 6: 307-312.

Cuetara BLV, Crotti TN, O'Donoghue AJ et al. 2006; Cloning and characterization of osteoclast precursors from the RAW264.7 cell line. In Vitro Cell Dev Biol Anim 42: 182-188.

Fanord F, Fairbairn K, Kim H et al. 2011; Bisphosphonate-modified gold nanoparticles: a useful vehicle to study the treatment of osteonecrosis of the femoral head. Nanotechnology 22(3): 1-11.

Faucheux C, Verron E, Soueidan A et al. 2009; Controlled release of bisphosphonate from a calcium phosphate biomaterial inhibits osteoclastic resorption in vitro. $J$ Biomed Mater Res A 89: 46-56.

Gkioni K, Leeuwenburgh SCG, Douglas TEL et al. 2010; Mineralization of hydrogels for bone regeneration. Tissue Eng B Rev 16: 577-585.

Heo DN, Ko WK, Bae MS et al. 2014; Enhanced bone regeneration with a gold nanoparticle-hydrogel complex. J Mater Chem B 2: 1584-1593.
Hogan HA, Ruhmann SP, Sampson HW. 2000; The mechanical properties of cancellous bone in the proximal tibia of ovariectomized rats. J Bone Miner Res 15: 284-292. Kettenberger U, Latypova A, Terrier A et al. 2015; Time course of bone screw fixation following a local delivery of Zoledronate in a rat femoral model - a micro-finite element analysis. $J$ Mech Behav Biomed Mater 45: 22-31.

Kettenberger U, Ston J, Thein E et al. 2014; Does locally delivered Zoledronate influence peri-implant bone formation? Spatiotemporal monitoring of bone remodeling in vivo. Biomaterials 35: 9995-10 006.

Kruth HS, Jones NL, Huang W et al. 2005; Macropinocytosis is the endocytic pathway that mediates macrophage foam cell formation with native low density lipoprotein. J Biol Chem 280: 2352-2360.

LeGeros RZ 2008; Calcium phosphate-based osteoinductive materials. Chem Rev 108: 4742-4753.

Lobo-Escolar A, Joven E, Iglesias D et al. 2010; Predictive factors for cutting-out in femoral intramedullary nailing. Injury 41: 1312-1316.

Martínez-Álvarez C, González-Meli B, BerenguerFroehner B et al. 2013; Injection and 
adhesion palatoplasty: a preliminary study in a canine model. $J$ Surg Res 183: 654-662.

Merrell MA, Wakchoure S, Ilvesaro JM et al. 2007; Differential effects of $\mathrm{Ca}^{2+}$ on bisphosphonate-induced growth inhibition in breast cancer and mesothelioma cells. Eur J Pharmacol 559: 21-31.

Monkkonen J, Taskinen M, Auriola SOK et al. 1994; Growth inhibition of macrophagelike and other cell types by liposomeencapsulated, calcium-bound, and free bisphosphonates in vitro. J Drug Target 2: 299-308.

Nageeb M, Nouh SR, Bergman K et al. 2012; Bone engineering by biomimetic injectable hydrogel. Mol Cryst Liquid Cryst 555: 177-188.

Nancollas GH, Tang R, Phipps RJ et al. 2006; Novel insights into actions of bisphosphonates on bone: differences in interactions with hydroxyapatite. Bone 38: 617-627.

Nejadnik MR, Yang X, Bongio M et al. 2014; Selfhealing hybrid nanocomposites consisting of bisphosphonated hyaluronan and calcium phosphate nanoparticles. Biomaterials 35: 6918-6929.

Oh N, Park JH 2014; Endocytosis and exocytosis of nanoparticles in mammalian cells. Int J Nanomed 9(suppl 1): 51-63.

Ong HT, Loo JSC, Boey FYC et al. 2008; Exploiting the high-affinity phosphonatehydroxyapatite nanoparticle interaction for delivery of radiation and drugs. $J$ Nanopart Res 10: 141-150.

Orriss IR, Key ML, Colston KW et al. 2009; Inhibition of osteoblast function in vitro by aminobisphosphonates. J Cell Biochem 106: 109-118.
Palazzo B, Iafisco M, Laforgia M et al. 2007; Biomimetic hydroxyapatite-drug nanocrystals as potential bone substitutes with antitumor drug delivery properties. Adv Funct Mater 17: 2180-2188.

Peter B, Gauthier O, Laib S et al. 2006; Local delivery of bisphosphonate from coated orthopedic implants increases implants mechanical stability in osteoporotic rats. $J$ Biomed Mater Res A 76: 133-143.

Rogers MJ, Gordon S, Benford HL et al. 2000; Cellular and molecular mechanisms of action of bisphosphonates. Cancer $\mathbf{8 8}$ (12, suppl): 2961-2978.

Roshan-Ghias A, Lambers FM, GholamRezaee $\mathrm{M}$ et al. 2011; In vivo loading increases mechanical properties of scaffold by affecting bone formation and bone resorption rates. Bone 49: 1357-1364.

Scheel J, Weimans S, Thiemann A et al. 2009; Exposure of the murine RAW 264.7 macrophage cell line to hydroxyapatite dispersions of various composition and morphology: assessment of cytotoxicity, activation and stress response. Toxicol In Vitro 23: 531-538.

Schulte FA, Lambers FM, Kuhn G et al. 2011; In vivo micro-computed tomography allows direct three-dimensional quantification of both bone formation and bone resorption parameters using time-lapsed imaging. Bone 48: 433-442.

Sörensen TC, Arnoldi J, Procter P et al. 2013; Locally enhanced early bone formation of zoledronic acid incorporated into a bone cement plug in vivo. J Pharm Pharmacol 65: 201-212.

Stromsoe K, Kok WL, Hoiseth A et al. 1993; Holding power of the $4.5 \mathrm{~mm} \mathrm{AO} / \mathrm{ASIF}$ cortex screw in cortical bone in relation to bone mineral. Injury 24: 656-659.

Suratwala SJ, Cho SK, van Raalte JJ et al. 2008; Enhancement of periprosthetic bone quality with topical hydroxyapatite-bisphosphonate composite. J Bone Joint Surg Am 90: 2189-2196.

Tami AE, Leitner MM, Baucke MG et al. 2009; Hydroxyapatite particles maintain periimplant bone mantle during osseointegration in osteoporotic bone. Bone 45: 1117-1124.

Thompson K, Rogers MJ, Coxon FP et al. 2006; Cytosolic entry of bisphosphonate drugs requires acidification of vesicles after fluid-phase endocytosis. Mol Pharmacol 69: 1624-1632.

Waarsing JH, Day JS, Van Der Linden JC et al. 2004; Detecting and tracking local changes in the tibiae of individual rats: a novel method to analyse longitudinal in vivo micro-CT data. Bone 34: 163-169.

Wang L, Zhang M, Yang Z et al. 2006; The first pamidronate containing polymer and copolymer. Chem Commun 26: 2795-2797.

Wilmes B, Rademacher C, Olthoff G et al. 2006; Parameters affecting primary stability of orthodontic mini-implants. $J$ Orofac Orthop 67: 162-174.

Wirth AJ, Goldhahn J, Flaig C et al. 2011; Implant stability is affected by local bone microstructural quality. Bone 49: 473-478.

Yoshinari M, Oda Y, Inoue Tet al. 2002; Bone response to calcium phosphate-coated and bisphosphonate-immobilized titanium implants. Biomaterials 23: 2879-2885.

Zhou H, Lee J 2011; Nanoscale hydroxyapatite particles for bone tissue engineering. Acta Biomater 7: 2769-2781. 